\title{
Bus Line Number Detection Employing MY VISION
}

\author{
Ye Zhou, Yosiki Hamasaki, Joo Kooi Tan \\ Department of Mechanical and Control Engineering, \\ Kyushu Institute of Technology, \\ Sensui-cho Tobata-ku \\ Kitakyushu, 804-8550, Japan \\ E-mail: \{zhou-ye, etheltan\}@ss10.cntl.kyutech.ac.jp \\ http://lab.cntl.kyutech.ac.jp/ etheltan/
}

\begin{abstract}
In response to the requests from visually impaired people that they wish better assistance tools in their daily activities, and to the facts that they find difficulty in using public transportation, we propose a bus boarding support system using MY VISION. For the support, we detect a bus approaching a bus stop from MY VISION images employing Haar-like filters, and acquire the moving object area with the pyramidal Lucas-Kanade method. Then, we extract the frontal upper area displaying bus line number and the destination by random forest. Having obtained the area, a method of connecting area marking is used to obtain the bus line number area. The effectiveness of the proposed method is shown by experiments.
\end{abstract}

Keywords: Bus line number, Moving area detection, Haar-like filters, Random forest, Visually impaired.

\section{Introduction}

According to the statistics survey from the Ministry of Labor in the past 23 years, there are about 320,000 people with visual impairment in Japan ${ }^{[1]}$. Meanwhile, about $69 \%$ of these people are over 65 years old. It can be seen that with the development of social aging, the number of people with visual impairment is further increasing. For the visually impaired, considering practicality and economic cost, visual impairment usually chooses public transportation. However, public transportation needs a lot of visual information and there are many interference problems, such as a bus. In addition, a bus also contains critical information of the line number and entrance. If the lack of the above information, the visually impaired cannot successfully complete the ride behavior. Therefore, it is necessary to develop a support system that enables visually impaired people to easily use public transportation facilities. In this paper, we propose a visual disability support system that can detect the frontal LED area of a bus and recognize its route number.

Although the computational cost of using learning results to detect specific moving objects in a complex image background is high, the detection results are accurate. This paper proposes a method of detecting a specific moving object. It extracts a moving object area by using the optical flow method, updates the color histogram of the area based on the Camshift ${ }^{[2]}$ tracking method, and corrects the tracking bounding box to complete the entire tracking and recognition process.

The system in this paper contains two structures: learning unit and recognition unit. (1) Learning unit. According to the input image, the Haar-like ${ }^{[3]}$ feature quantity is calculated and extracted, which is based on the brightness difference between the two regions and is robust to noise and brightness changes. After that, the random forest classification method is used to effectively classify the feature quantities. (2) Identification unit. The bus LED area recognition is performed using Harris angle detector to extract the feature points of the input image, and the pyramidal Lucas-Kanade optical flow algorithm ${ }^{[4]}$ to obtain the moving object area ${ }^{[5]}$. After that, Random Sample Consensus (RANSAC) ${ }^{[6]}$ is used to filter out the interference optical flow information generated by the camera movement, and then use the $k$ means clustering method to obtain the target area. In the clustering area, the learning-based recognition method is used to complete the task of bus LED area recognition. After a certain target area is obtained, the tracking task is performed.

\section{Related work}

In the literature, there are many automatic bus number recognition systems. However, most of them use active sensors, such as GPS tracking systems, RFID, etc. 
For vision-based methods, Guida ${ }^{[7]}$ proposed the detection and identification of bus line numbers ${ }^{[8]}$. Their method uses Adaboost-based cascaded classifiers to locate the main geometric entities of the bus facade, and the matching is improved by robust geometric matching. Then the bus façade image is converted to a HSV color space and threshold operations are applied to segment each number.

Pan [9] proposed another vision-based method that detects information from the front of the bus. Their system can be divided into 2 subsystems: bus detection and bus line number detection. In the bus detection, they use HOG and SVM to detect the bus position. In the bus line number detection, they use adjacent character grouping and sophisticated edge detection to find candidate regions. The Haar-like feature is extracted from edge distribution. The acquired features are input into Adaboost to classify each component. Finally, they combine an optical character recognition software with a text-to-speech synthesizer to produce audio.

In short, compared with the aforementioned tracking and recognition method, computational complexity of their methods is high, and the recognition accuracy and the effect of tracking moving objects still need to be improved. Therefore, in the feature area selection, the proposed method uses the optical flow method to effectively filter out the moving object area. At the same time, the Camshift tracking method used in this article has low computational complexity and has certain practicability.

\section{Learning unit}

\subsection{Haar-like feature}

For the detection of a bus LED area, this article is divided into two types of images for feature learning: the bus LED area and the background area. In order to unify the input image size, it is standardized to $100 \times 30$ pixels. In the previous methods based on feature learning, the most commonly used method for vehicle feature learning is HOG feature representation. However, when the feature shapes of two regions are similar, the HOG feature is prone to representation errors, which will affect the subsequent recognition tasks. Therefore, in this article, it is proposed to use Haar-like features for regional characterization. Its main advantage is that it characterizes not only the shape of the object, but also the brightness distribution of the image area effectively. Figure 1 shows an example diagram of the Haar-like filter and calculation is done by the following formula;

$$
H(A, B)=F(A)-F(B)
$$

Here, $F(A)$ and $F(B)$ are the average brightness of area $A$ and $B$.

$$
\text { A } B
$$

As shown in Fig. 2, the proposed method uses 6 different Haar-like filters. The 6 Haar-like features

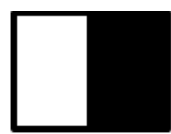

Fig. 1 Haar feature

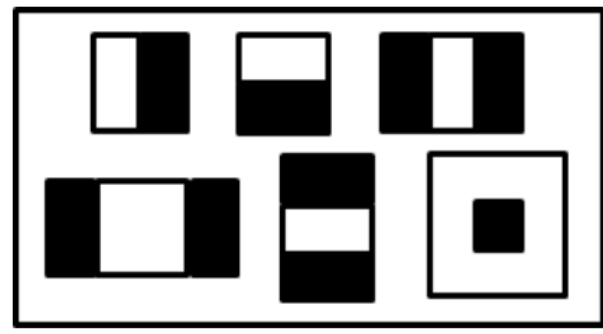

Fig. 2 Six types of filters

include two edge features, three linear features and the center-around feature. After obtaining the Haar-like features of the LED area, it is necessary to classify and learn the obtained features. Random forest is used as a classification method in the proposed method.

\subsection{Random forest}

The system proposed in this paper, it needs to complete the task of identifying multiple types of targets. Therefore, random forest learning ${ }^{[10]}$ meets the needs of this method. It creates a feature tree with low correlation by selecting feature quantities at the non-terminal nodes of each decision tree. It calculates the Haar-like feature value of each input image to create a sample. A subset is randomly generated from the sample, and a random decision tree is created using the branch function of each subset to perform learning. The random forest is determined by inputting the feature vector of the unknown sample into the decision tree. It calculates the category distribution of the terminal node that finally reaches the unknown sample, and uses this result to estimate the category of the unknown sample through the following equation; 


$$
P(c / S)=\frac{1}{T} \sum_{t=1}^{T} P_{t}(c / S)
$$

In the above formula, $T$ is the number of trees in the random forest, and $P(c / S)$ is the class distribution.

\section{Recognition}

The target recognition task is divided into two subtasks: bus LED area recognition and bus line number recognition.

\subsection{Bus LED area recognition}

In order to reduce the amount of data processing, the optical flow method is used to obtain a moving object area ${ }^{[10]}$ to achieve a faster target recognition task. The calculation process is as follows:

1) Use Harris angle detector to acquire the feature points of the target and establish a moving target area. After the feature points are detected, take $r$ frames as the interval. The moving object area is obtained by using the LK pyramid optical flow and k-means clustering method.

2) Since the moving object area obtained by the optical flow method usually includes the noise caused by the camera movement, it is necessary to use the projection transformation model to estimate the camera movement and remove the noise area generated by it. The calculation formula of this model is as follows;

$$
\left\{\begin{array}{l}
x_{2}=\frac{h_{0} x_{1}+h_{1} y_{1}+h_{2}}{h_{6} x_{1}+h_{7} y_{1}+1} \\
y_{2}=\frac{h_{3} x_{1}+h_{4} y_{1}+h_{5}}{h_{6} x_{1}+h_{7} y_{1}+1}
\end{array}\right.
$$

Use RANSAC to determine the location parameters $h_{i}(i=0,1 \ldots 7)$ of Eq. (3). The RANSAC can effectively divide the internal optical flow area of moving objects and the external optical flow of noise generated by camera movement. Then use the random forest to complete the recognition of the bus LED area.

\subsection{Bus line number recognition}

After obtaining the LED area, it needs to be further processed to obtain the digital area.
According to the relatively fixed position distribution of the LED display area of Japanese buses, a more accurate line number area can be obtained. Then use HSV color processing to obtain the binary image of the digital part, and use the connected area labeling method to segment the digital area.

\section{Experimental results}

\subsection{Setup}

In this section, we show the results of the method proposed in this paper to learn the characteristics of the bus LED area, as well as the recognition results of the specific moving target area and the line number recognition results obtained from object tracking.

The learning results of the random forests are evaluated by test data. At the same time, the accuracy of the bus LED area detection is also evaluated.

Table 1 shows detailed parameter values for the learning of the random forest. Among them, the dimension of Haar-like features is 5102 .

Table 1 Learning parameters of the random forest.

\begin{tabular}{ccc}
\hline Learning images & Positive image: & 1200 \\
& Negative image: & 800 \\
\hline Test images & Each class: & 500 \\
Size & $100 \times 30$ & \\
Number of subsets & 30 & \\
Tree depth & 5 & \\
Feature selection times & 30 & \\
Threshold selection times & 30 \\
\hline
\end{tabular}

\subsection{Results}

For the learning results of random forest, we use the classification accuracy to evaluate the performance for the plate of bus and the background.

Table 2 shows the result of the classification. It proves that the proposed method can achieve a good performance for the task of classification. Figure 3 shows the detection performance of the method.

Table 2 Random forest learning results 


\begin{tabular}{ccc}
\hline & Plate & Background \\
\hline Plate & $90.4 \%$ & $9.6 \%$ \\
Background & $4.0 \%$ & $96.0 \%$ \\
\hline
\end{tabular}

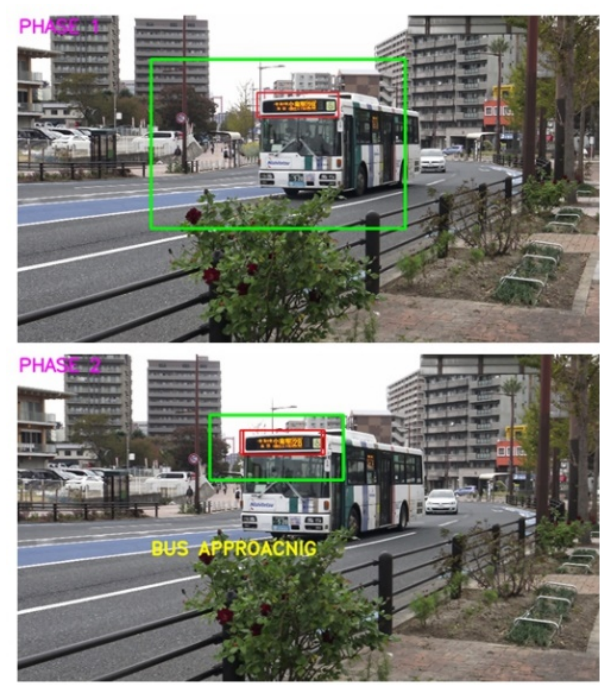

(a)

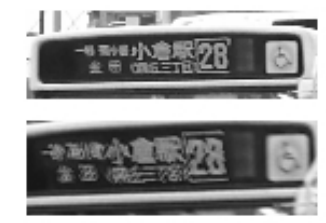

(b)

Figure 3 Examples of detection result for the bus LED and the line number

The average processing time of each picture is 1.56 [s], and the success rate of detecting the LED panel area in the window of the moving object is $91.3 \%$.

\section{Discussion and conclusion}

This paper proposes a bus travel assistance system for the visually impaired. The system includes bus LED area recognition and line number recognition tasks. Among them, the overall system includes learning method design, area tracking method design and recognition method design. The final experimental results prove the effectiveness of the proposed method and the practicability of the system.

In the course of the experiment, it was found that when identifying the LED area, there was misrecognition due to lighting conditions, and interference from moving cars.
Therefore, in future work, it is necessary to expand the existing data set and adjust the learning parameters to obtain higher recognition accuracy.

\section{References}

1. Ministry of Health, Labor and Welfare, Social Assistance Bureau, Disability Health and Welfare Department: 2011 Survey on Difficulty in Living (Fact-finding Survey on Children at Home, etc.) Results, Ministry of Health, Labor and Welfare, Social Assistance Bureau, Disability Health and Welfare Department, 2013, pp.13.

2. O.-D. Nouar, G. Ali, C. Raphael Canals. Improved object tracking with camshift algorithm, IEEE International Conference on Acoustics Speech and Signal Processing, 2006, pp.36-42.

3. Gong Haifan, Chen Limin, Li Changhao, Wang Yue. Online tracking and relocation based on a new rotationinvariant Haar-Like statistical descriptor in endoscopic examination, IEEE Access, 99, 2020, pp.69-83.

4. Dowson Nicholas, Bowden Richard. Mutual information for Lucas-Kanade tracking, An inverse compositional formulation, IEEE Transactions on Pattern Analysis and Machine Intelligence, 30(1), 2008, pp.180-185.

5. Ishitobi K. Tan J K. Kim H. Detection of a specific moving object from head-mounted camera images. IEEE International Symposium on System Integration (SII), 2017, pp. 6pages.

6. M.A. Fischler, R.C. Bolles. Random Sample Consensus: a paradigm for model fitting with applications to image analysis and automated cartography. The ACM Communication, 1981, pp.381-395.

7. C. Guida, D. Comanducci, C. Colombo. Automatic bus line number localization and recognition on mobile phones. A computer vision aid for the visually impaired, Image Analysis and Processing (ICIAP), 2011, pp. 323-332.

8. Pitchakorn Wongta. An automatic bus route number recognition. International Joint Conference on Computer Science and Software Engineering (JCSSE), 2016, pp.1924.

9. H. Pan, C. Yi, Y. Tian. A primary travelling assistant system of bus detection and recognition for visually impaired people, IEEE International Conference on Multimedia and Expo Workshops (ICMEW), 2013, pp. 16.

10. F.Breiman. Random forest. Machine Learning, Vol, 45, No. 1, 2001, pp. 5-32. 